Sub-Surface Characterization and Three Dimensional Profiling of Semiconductors by Magnetic Resonance Force Microscopy

Author(s):

Submitted to:
P. Chris Hammel, MST-10

Gregory Moore, CST-4

Michael Roukes, California Institute of Technology

Zhenyong Zhang, MST-10
RECENES

OCT 11 १९SS

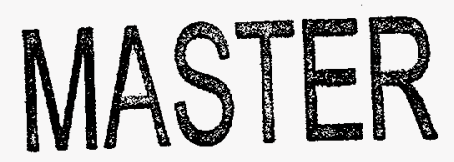

Los Alamos

NATIONAL LABORATORY
DOE Office of Scientific and Technical Information (OSTI)

DISTRIBUTION OF THIS DOCUMENT IS UNLMITED

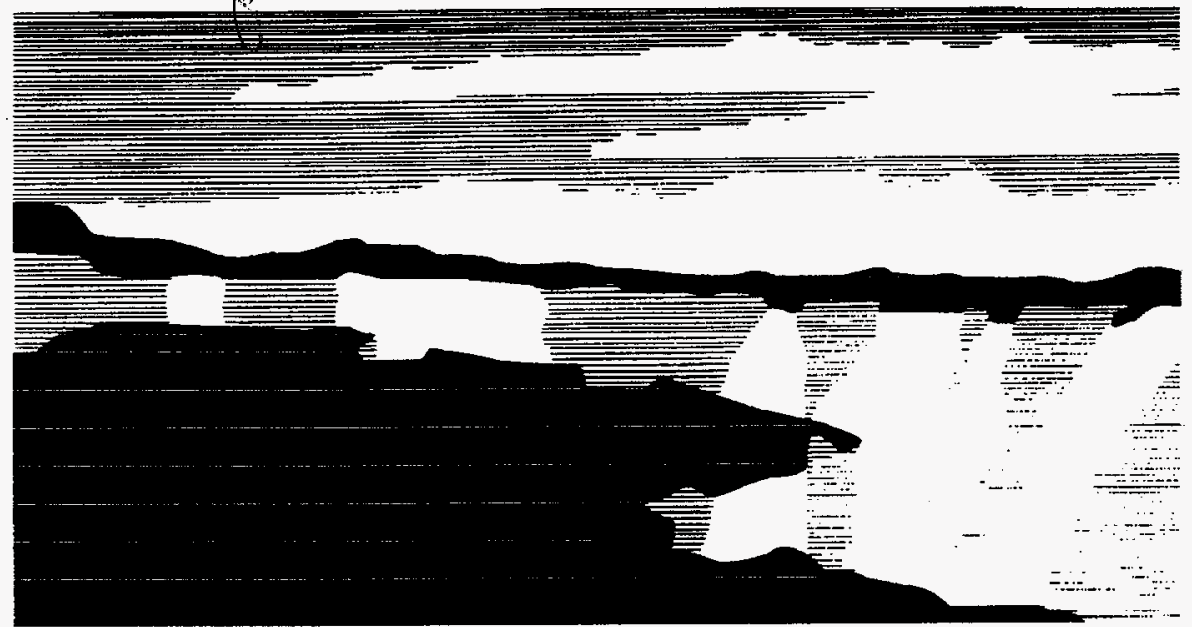

Los Alamos National Laboratory, an affirmative action/equal opportunity employer, is operated by the University of California for the U.S. Department of Energy under contract W-7405-ENG-36. By acceptance of this article, the publisher recognizes that the U.S. Government retains a nonexclusive, royaltyfree license to publish or reproduce the published form of this contribution, or to allow others to do so, for U.S. Government purposes. The Los Alamos National Laboratory requests that the publisher identify this article as work performed under the auspices of the U.S. Department of Energy. 


\section{DISCLAIMER}

Portions of this document may be illegible in electronic image products. Images are produced from the best available original document. 


\title{
Sub-Surface Characterization and Three Dimensional Profiling of Semiconductors by Magnetic Resonance Force Microscopy
}

\author{
P. Chris Hammel*, Gregory Moore, Michael Roukes (California Institute of \\ Technology), and Zhenyong Zhang
}

\begin{abstract}
This is the final report of a two-year, Laboratory-Directed Research and Development (LDRD) project at the Los Alamos National Laboratory (LANL). The project successfully developed a magnetic resonance force microscope (MRFM) instrument to mechanically detect magnetic resonance signals. This technique provides an intrinsically subsurface, chemical-species-specific probe of structure, constituent density and other properties of materials. As in conventional magnetic resonance imaging (MRI), an applied magnetic field gradient selects a well defined volume of the sample for study. However mechanical detection allows much greater sensitivity, and this in turn allows the reduction of the size of the minimum resolvable volume. This requires building an instrument designed to achieve nanometer-scale resolution at buried semiconductor interfaces. High-resolution, three-dimensional depth profiling of semiconductors is critical in the development and fabrication of semiconductor devices. Currently, there is no capability for direct, highresolution observation and characterization of dopant density, and other critical features of semiconductors. The successful development of MRFM in conjunction with modifications to improve resolution will enable for the first time detailed structural and electronic studies in doped semiconductors and multilayered nanoelectronic devices, greatly accelerating the current pace of research and development.
\end{abstract}

\section{Background and Research Objectives}

Modern electronics research and development has recently focused on the study of sub-micron scale structures and devices such as semiconductor heterojunctions, semiconductor-metal interfaces, quantum wires, quantum wells, quantum dots, molecular transistors and semiconductor films. However, no technology currently exists which allows for direct

* Principal investigator, e-mail: pch@lanl.gov 
observation and characterization of buried interfaces, defects, dislocations or impurities that critically effect device operation.

The magnetic resonance force microscope (MRFM) is a microscopic imaging instrument based on a recent novel proposal [1-3] to mechanically detect magnetic resonance signals. The spin magnetization $m$ of a sample is detected by means of the force $F=(m \bullet \nabla) B$ between $m$ and the magnetic field gradient $\nabla \mathrm{B}$ generated by a permanent magnet. This force is sensitively measured by laser interferometric detection of the motion of a micro-mechanical resonator. This approach to detection of both electron spin and nuclear magnetic resonance has been successfully demonstrated [4-5]. In conjunction with the sharp spectral response of the magnetic resonance technique, the field gradient of the permanent magnet also enables high resolution spatial imaging as in conventional magnetic resonance imaging (MRI). This field gradient allows identification of magnetic resonance signals arising from different spatial locations.

All experiments to date, including those we report here, have been performed with the sample mounted on the cantilever. The sample is then placed in the field of a permanent magnet mounted on a translatable stage. An rf field generated by a nearby coil is used to periodically modulate the force on the cantilever by modulating $m$. Only those spins having a resonance frequency $f=\gamma B(z)$ matching the rf frequency are manipulated, so only these contribute the time dependent force. This manipulation, by magnetic resonance techniques, is used to produce a periodic force at the characteristic frequency, $f_{C}$, of the cantilever (of order tens of $\mathrm{kHz}$ for commercial cantilevers) which drives its oscillation.

\section{Importance to LANL's Science and Technology Base and National R\&D Needs}

This project supports Los Alamos core competencies in nuclear and advanced materials as well as complex experimentation and measurement. It also enhances the Laboratory's capabilities in nanotechnology, national security, and industrial competitiveness. In nanotechnology, one of the most promising frontiers in advanced materials is the burgeoning field of nanostructures, self-assembled structures, quantum devices and nanophase materials. A key requirement for developing these technologies is the availability of a high resolution subsurface imaging and characterization tool. MRFM is the only technology able to fill this requirement. High performance electronic devices are crucial elements of our defense arsenal. Bringing the capabilities of the MRFM to bear will produce unprecedented advances in investigations of buried features of heterostructures and greatly accelerate the pace of research and development. The development of the MRFM will contribute to US industrial 
competitiveness by filling two crucial needs. It will enhance the ability of the semiconductor industry to model devices and provide a tool for process control on the device fabrication line. The MRFM instrument itself will be a product which can be marketed by companies who supply characterization equipment (STM and AFM etc.) to the semiconductor industry, such as Digital Instruments and Advanced Micro Devices.

\section{Scientific Approach and Results to Date}

We presently use commercially available [6] atomic force microscope cantilevers (i.e. clamped on one end only) with force constants of approximately $0.08 \mathrm{~N} / \mathrm{m}$ (unloaded) and resonance frequencies of approximately $6-20 \mathrm{kHz}$. We mount small (approximately 2-20 nanogram) samples of an organic molecule, diphenylpicrylhydrazyl (DPPH), which contains a single unpaired electronic spin on the cantilever tip. This mass reduces the resonance frequency (and $Q$ ) of the resonator. The $Q$ of loaded cantilevers can be as high as 30,000 in vacuum at room temperature. $\mathrm{A} \mathrm{NdFeB}$ magnet $(0.56 \mathrm{~mm}$ diameter by $6.35 \mathrm{~mm}$ long) which provides the field gradient is mounted on a translatable stage. We also apply a spatially homogeneous magnetic field which can be simultaneously ramped and modulated: $B=B_{o}(t)+$ $B_{\text {mod }} \cos \left(2 \pi f_{\text {mod }} t\right) ; f_{\text {mod }}=2-50 \mathrm{kHz}$. The homogeneous field is parallel to $\nabla B$ and these define the orientation of the $z$-axis. The field at the sample is the sum of the ramp, modulation and bar magnet fields. The oscillation of the cantilever is detected by means of a fiber-optic, diode-laser interferometer that has a sensitivity of $0.002 \AA / \mathrm{Hz}^{1 / 2}$. The optical fiber end is within a few microns of the cantilever. A small coil placed near the sample allows the application of radio frequency field $B_{1}$ (frequency $f_{\mathrm{rf}}=500-1000 \mathrm{MHz}$ ) to saturate the electron spin magnetization. The force sensitivity of the instrument is limited by thermal excitations of the cantilever described by a thermal noise force [7] $F_{\mathrm{n}}=\left(2 k k_{B} T \Delta v / \pi Q f_{c}\right)^{1 / 2}$ which depends on the cantilever properties: spring constant $k$, characteristic frequency $f_{c}$, and quality factor $\mathbf{Q}$, as well as the detection bandwidth $\Delta v$, and temperature $T$.

In Figure 1, we show the signal from an approximately $5 \mathrm{ng}$ particle containing $8 \times 10^{12}$ spins obtained by anharmonic modulation which displays the expected derivative line shape. For this signal $f_{\text {mod }}=26.38 \mathrm{kHz}, f_{1}=38.42 \mathrm{kHz}$ and $f_{\mathrm{c}}=12.04 \mathrm{kHz}$. If $m(t)$ is the (time dependent) particle magnetization, the magnitude of the force component at the cantilever frequency is given by $F\left(f_{c}\right)=\int(m(t) \bullet \nabla B) \sin \left(2 \pi f_{c} t\right) d t$. This force depends on several factors including the $z$-dependence of the spin density (i.e. along the direction of $\nabla B$ ), $B_{\text {mod, }}$, and the intrinsic line width, $\Delta B_{\mathrm{lw}}$. As the field is modulated, the position of the resonance field $B_{\text {Larmor }}=f_{\mathrm{rf}} / \gamma$ oscillates in the $z$-direction with amplitude $\delta_{\bmod }=B_{\bmod } / \nabla B \mid$ Analogously we define $\delta_{\mathrm{lw}}=\Delta B_{\mathrm{lw}} /|\nabla B|$ 
Numerical evaluation of this integral shows that for a particle of radius $R$, the maximum signal is obtained for $\delta_{\text {mod }} \cong R$. In this case $F\left(f_{c}\right) \approx 0.3 M_{z^{z}} \nabla B \mid \pi^{1 / 2}\left(\delta_{l w} / R\right)$, where $M_{z}$ is the total magnetization of the particle. In our case $B_{\text {mod }} \approx 3-5$ Gauss, $|\nabla B|=0.8 \mathrm{G} / \mu \mathrm{m}$, and $R \sim 9 \mu \mathrm{m}$, so $\delta_{\text {mod }} \cong R / 2$. Thus we expect the signal force to be $3 \times 10^{-15} \mathrm{~N}\left(f_{\mathrm{Larmor}}=709\right.$ $\mathrm{GHz}, \gamma=2.8 \mathrm{GHz} / \mathrm{kG}$ ). The calculated thermal noise force (room temperature, $\mathrm{Q}=3 \times 10^{4}, f_{\mathrm{c}}$ $\left.=1.2 \times 10^{4}, k=0.08 \mathrm{~N} / \mathrm{m}\right)$ is $\sim 4.1 \times 10^{-16} \mathrm{~N}$. This is larger than, but in agreement with, the observed noise given the uncertainties in various parameters describing the cantilever. The expected signal-to-noise ratio then should then be $\sim 10$, which agrees well with the observation.

To demonstrate the ability to image a sub-surface volume of magnetization, we placed two $\sim 15 \mu \mathrm{m}$ particles on the $\sim 30-40 \mu \mathrm{m}$ wide cantilever, one above and one below. The signal obtained from this sample is shown in Figure 2. The data were taken as a function of field $B_{\mathrm{O}}$. As we will discuss below, we know $B_{\mathrm{bar}}(z)$. From this and the resonance frequency we can plot the data as a function of distance between the cantilever and end of the bar magnet. The thickness of the silicon cantilever is $\sim 1-3 \mu \mathrm{m}$. The signals from the two particles are clearly distinguishable although not entirely distinct. The overlap of the two signals arises because $\delta_{\text {mod }}$ exceeds the spacing between the particles. This resolution can be improved by reducing $B_{\text {mod }}$ but at the expense of signal.

In Figures 3 and 4, we characterize the sensitivity of the MRFM to the position of the particle relative to the bar magnet. The fundamental parameter determining the resolution is the magnitude of the field gradient. We obtain this from the results shown in Figure 3. This shows the results of several measurements taken as the bar magnet is translated toward the sample. The field experienced by the sample is inferred in our measurements from knowledge of the resonance frequency $f_{\mathrm{rf}}$ and the homogeneous background field $B_{\mathrm{o}}: B_{\mathrm{bar}}=\left(f_{\mathrm{rr}} / \gamma\right)-B_{\mathrm{o}}$. The (position dependent) field gradient of the magnet is approximately $0.8 \mathrm{G} / \mu \mathrm{m}=80 \mathrm{~T} / \mathrm{m}$.

One can take advantage of this magnet geometry to improve sensitivity in studies of two-dimensional systems which are uniform in the transverse direction but for which high resolution in the $z$-direction is desired. The vanishing lateral gradient provides a thin, diskshaped "sensitive" volume which would include a large area of spin magnetization while maintaining high vertical resolution.

The heart of the MRFM is the micromechanical resonator. Miniaturization of this resonator is key to obtaining optimum instrument resolution and sensitivity. To date, we have fabricated monocrystalline, submicron-scale resonant beams with resonant frequencies in the vicinity of $100 \mathrm{MHz}$ from both $\mathrm{Si}$ and from GaAs. To date, miniature Ni columns of dimensions approximately $80 \mathrm{~nm}$ diameter $\times 500 \mathrm{~nm}$ high have been produced in our laboratory. 
This work has been the subject of an invited talk and has been described in the peerreview literature [9].

\section{References}

[1] J.A. Sidles, Appl. Phys. Lett. 58, 2854 (1991).

[2] J.A. Sidles, Phys. Rev. Lett. 68, 1124 (1992).

[3] J.A. Sidles, J.L. Garbini, and G.P. Drobny, Rev. Sci. Instrum. 63, 3881 (1992).

[4] D. Rugar, C.S. Yannoni, and J.A. Sidles, Nature 360, 563 (1992).

[5] D. Rugar, O. Züger, S. Hoen, C.S. Yannoni, H.-M. Vieth, and R.D. Kendrick, Science 264, 1560 (1994).

[6] Digital Instruments, 520 E. Montecito St, Santa Barbara, CA. The experiments reported here use type ESP cantilevers which are $450 \mu \mathrm{m}$ long, 30-40 $\mu \mathrm{m}$ wide and 1-3 $\mu \mathrm{m}$ thick.

[7] J.A. Sidles and D. Rugar, Phys. Rev. Lett. 70, 3506 (1993).

[8] K.J. Bruland, J. Krzystek, J.L. Garbini, and J.A. Sidles, preprint.

[9] P.C. Hammel, Z. Zhang, G.J. Moore, and M.L. Roukes, J. Low Temp. Physics, 101, 59 (1995). 


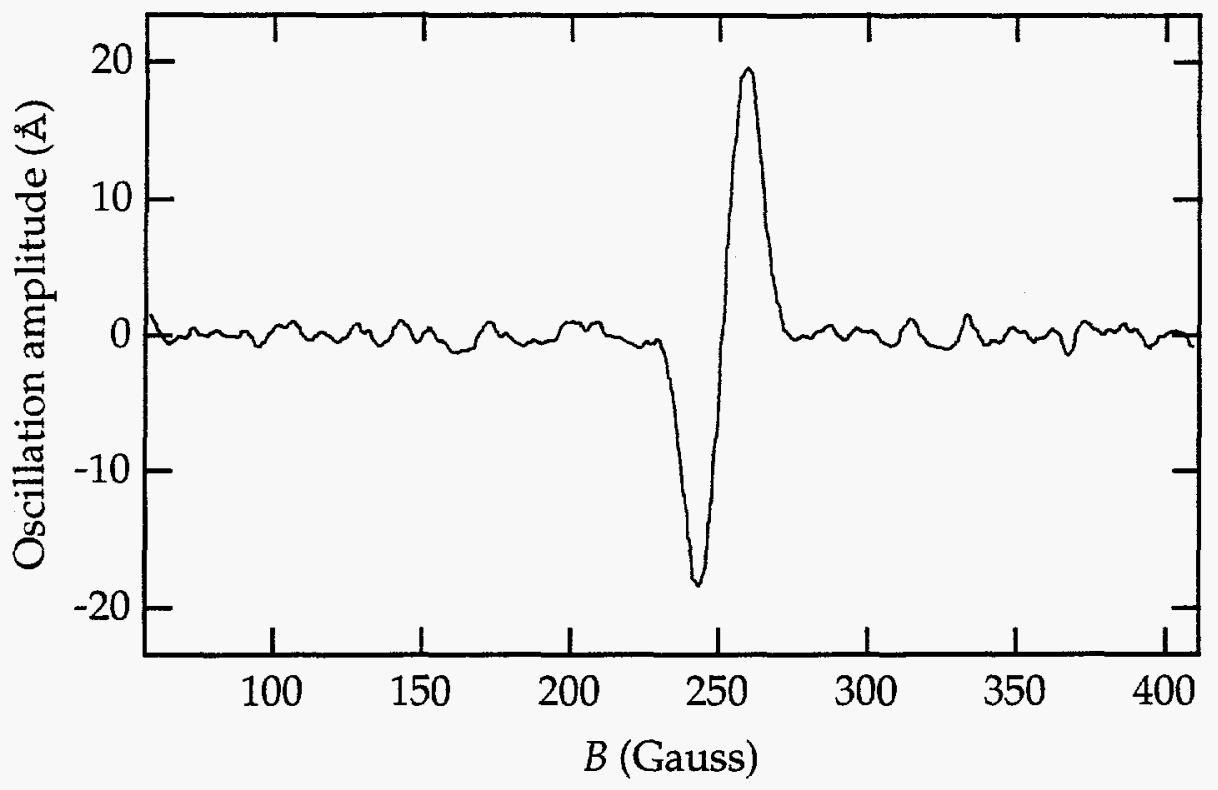

Fig. 1. Single shot MRFM signal from a $\sim 18 \mu \mathrm{m}$ diameter $(5 \mathrm{ng})$ particle of DPPH: the amplitude of the cantilever oscillation is shown as a function of the applied homogeneous field $B=B_{\mathrm{bar}}+B_{\mathrm{o}}$. The $\mathrm{rf}$ frequency is $709 \mathrm{MHz}$, thus at resonance the total field is $253 \mathrm{G}$. 


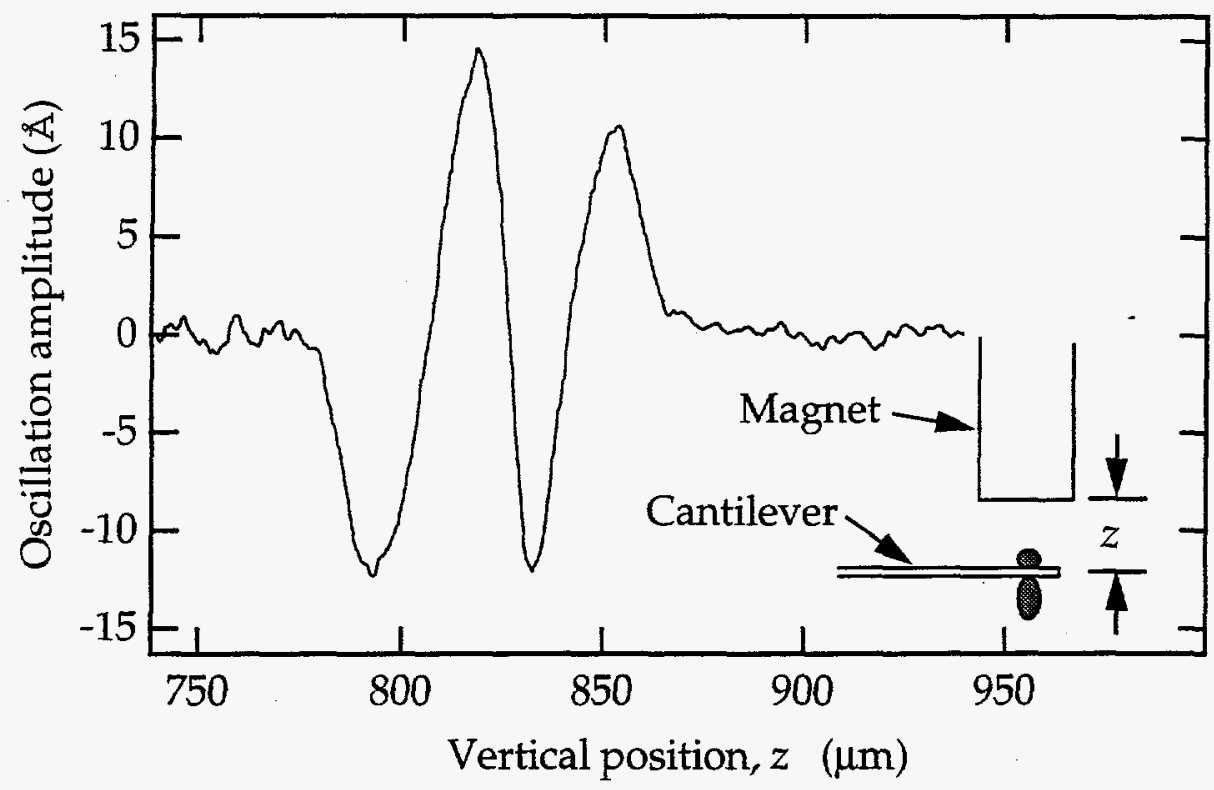

Fig. 2. The swept field MRFM signal from two particles of DPPH, one mounted above ( 7 $\mathrm{ng})$ and one below $(\sim 3 \mathrm{ng})$ the cantilever. Signal intensity is shown as a function of distance, $z$, from the end of the bar magnet to the cantilever. Signal was obtained at a $f_{\mathrm{rf}}=828 \mathrm{MHz}$. The distance, $z$, is deduced from knowledge of $B_{\text {Larmor }}, B_{0}$, and $B_{\mathrm{bar}}(z)$. A schematic (not to scale) representation of the geometry is shown in the inset. 


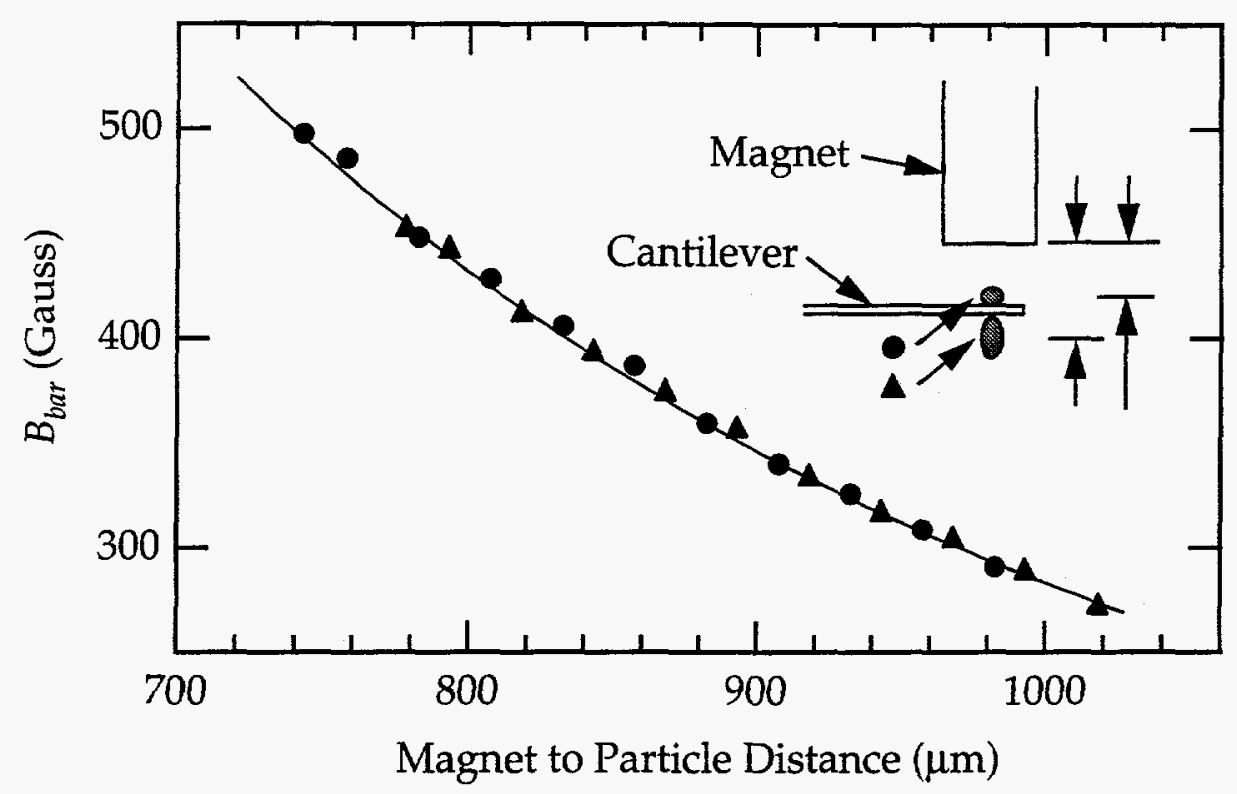

Fig. 3. The magnitude of the field due to the bar magnet $\left(B_{\mathrm{bar}}=B_{\mathrm{Larmor}}-B_{\mathrm{o}}\right)$ at the center of each of the two particles (upper particle, solid circles; lower particle, solid triangles) is shown as a function of the distance between the end of the magnet and the center of the particles. The solid line is a fit to the expected dependence of field on distance from the end of a cylindrical magnet along its axis. From this fit, the separation between the centers of the two particles is found to be $35.4( \pm 1) \mu \mathrm{m}$. 


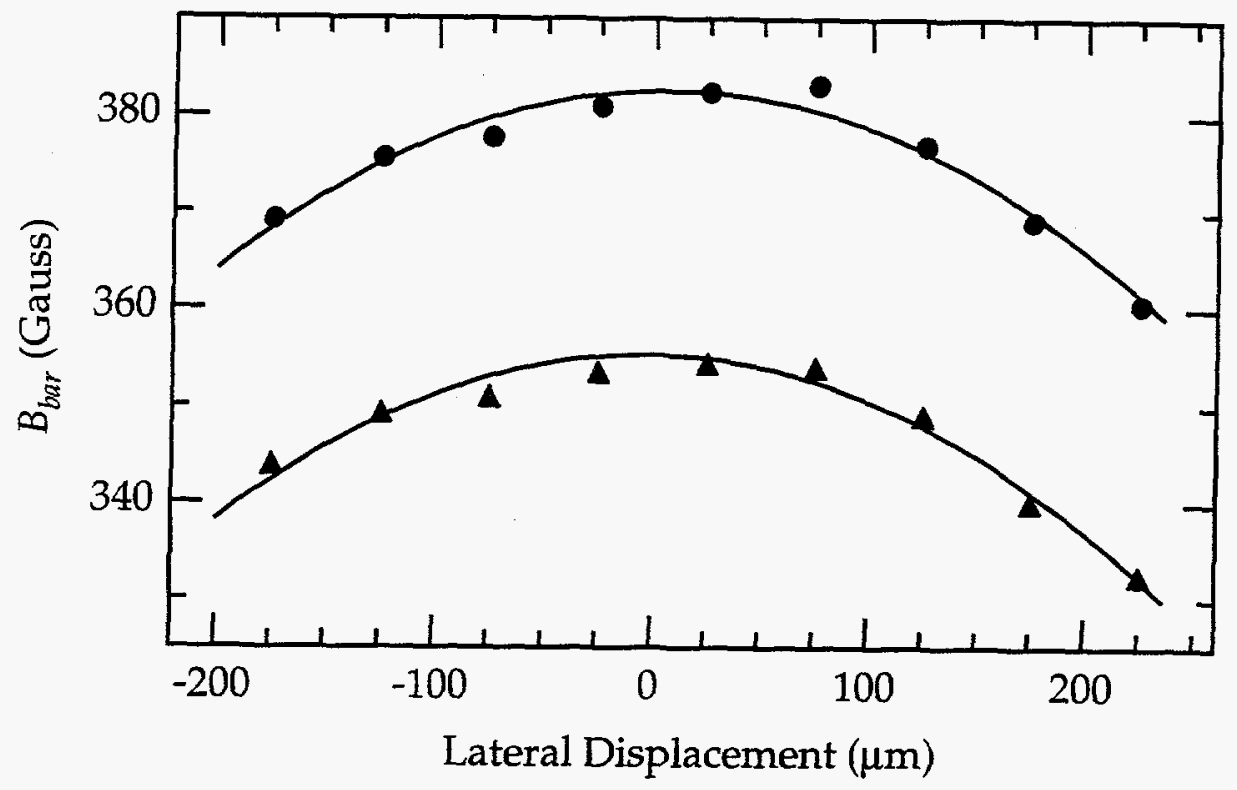

Fig. 4. Variation of the magnetic field of the bar magnet at the center of the DPPH particle as its position relative to the bar magnet is varied in a direction perpendicular to the bar axis.

\section{DISCLAIMER}

This report was prepared as an account of work sponsored by an agency of the United States Government. Neither the United States Government nor any agency thereof, nor any of their employees, makes any warranty, express or implied, or assumes any legal liability or responsibility for the accuracy, completeness, or usefulness of any information, apparatus, product, or process disclosed, or represents that its use would not infringe privately owned rights. Reference herein to any specific commercial product, process, or service by trade name, trademark, manufacturer, or otherwise does not necessarily constitute or imply its endorsement, recommendation, or favoring by the United States Government or any agency thereof. The views and opinions of authors expressed herein do not necessarily state or reflect those of the United States Government or any agency thereof. 